PROCEEDINGS OF THE

AMERICAN MATHEMATICAL SOCIETY

Volume 138, Number 5, May 2010, Pages 1739-1750

S 0002-9939(10)10203-2

Article electronically published on January 19, 2010

\title{
RESTRICTED SCHUR MULTIPLIERS AND THEIR APPLICATIONS
}

\author{
TIMUR OIKHBERG
}

(Communicated by Nigel J. Kalton)

\begin{abstract}
We compute the norm of the restriction of a Schur multiplier, arising from a multiplication operator, to a coordinate subspace. This result is used to generalize Wielandt's minimax inequality. Furthermore, we compute various $s$-numbers of an elementary Schur multiplier and determine criteria for membership of such multipliers in certain operator ideals.
\end{abstract}

\section{INTRODUCTION}

Throughout this paper, we work with unconditional matrix spaces. A Banach space $\mathfrak{X}$ of $I \times I$ matrices (where $I$ is a finite or infinite index set) is called an unconditional matrix space if:

(1) All matrices with finitely many non-zero entries belong to $\mathfrak{X}$.

(2) For any matrix $\left(a_{i j}\right) \in \mathfrak{X},\left\|\left(a_{i j}\right)\right\|=\sup _{J \subset I,|J|<\infty}\left\|\mathcal{P}_{J}\left(a_{i j}\right)\right\|$, where $\mathcal{P}_{J}$ is the truncation onto $J \times J$, that is, $\left(\mathcal{P}_{J}\left(a_{i j}\right)\right)_{p q}=\left\{\begin{array}{ll}a_{p q} & (p, q) \in J \times J \\ 0 & (p, q) \notin J \times J\end{array}\right.$.

(3) For any matrix $\left(a_{i j}\right) \in \mathfrak{X}$ and any two sequences $\left(\alpha_{i}\right)$ and $\left(\beta_{j}\right)$ of scalars of absolute value $1,\left\|\left(a_{i j}\right)\right\|=\left\|\left(\alpha_{i} \beta_{j} a_{i j}\right)\right\|$.

Clearly, tensor products of Banach spaces with 1-unconditional bases are unconditional matrix spaces. Another important class of examples arises in the following way. Suppose $\mathcal{E}$ is a symmetric sequence space, that is, a Banach space of sequences such that, for any $\left(x_{1}, x_{2}, \ldots\right) \in \mathcal{E}$, we have:

(1) For any sequence $\left(\omega_{i}\right)$ in the unit ball of $\mathbb{C}$ and any permutation $\pi: \mathbb{N} \rightarrow \mathbb{N}$, $\left(\omega_{1} x_{\pi(1)}, \omega_{2} x_{\pi(2)}, \ldots\right) \in \mathcal{E}$ and $\left\|\left(\omega_{1} x_{\pi(1)}, \omega_{2} x_{\pi(2)}, \ldots\right)\right\|_{\mathcal{E}}=\left\|\left(x_{1}, x_{2}, \ldots\right)\right\|_{\mathcal{E}}$.

(2) $\left\|\left(x_{1}, x_{2}, \ldots\right)\right\|_{\mathcal{E}}=\lim _{n}\left\|\left(x_{1}, \ldots, x_{n}\right)\right\|_{\mathcal{E}}$, where we define $\left\|\left(x_{1}, \ldots, x_{n}\right)\right\|_{\mathcal{E}}$ as $\left\|\left(x_{1}, \ldots, x_{n}, 0,0, \ldots\right)\right\|_{\mathcal{E}}$.

Denote by $\mathcal{S}^{\mathcal{E}}$ the space of operators $T$ on $\ell_{2}$ whose sequences of singular values $\left(s_{i}(T)\right)_{i \in \mathbb{N}}$ belong to $\mathcal{E}$ (see Section II.7 of 12 for the definition of singular numbers for non-compact operators). The norm on this space is given by $\|T\|_{\mathcal{E}}=\left\|\left(s_{i}(T)\right)_{i \in \mathbb{N}}\right\|_{\mathcal{E}}$. By e.g. Chapters 1-2 of [28 or Chapter III of [12, this is an unconditional matrix space. Moreover, any separable symmetrically normed

Received by the editors May 3, 2009, and, in revised form, September 2, 2009.

2000 Mathematics Subject Classification. Primary 47B06, 47B10, 47B49, 47L20.

Key words and phrases. Schur product, elementary operators, minimax inequalities, $s$ numbers, operator ideals.

The author is grateful to I. Todorov for many stimulating conversations.

(C)2010 American Mathematical Society 
ideal is of the form $\mathcal{S}^{\mathcal{E}}$, where $\mathcal{E}$ is regular: $\lim _{n}\left\|\left(0, \ldots, 0, x_{n}, x_{n+1}, \ldots\right)\right\|_{\mathcal{E}}=0$ for any $\left(x_{1}, x_{2}, \ldots\right) \in \mathcal{E}$.

For the sake of brevity, we write $\mathcal{S}^{p}=\mathcal{S}^{\ell^{p}}$ for $1 \leqslant p<\infty$ and $K\left(\ell_{2}\right)=\mathcal{S}^{\infty}=\mathcal{S}^{c_{0}}$. The $n$-dimensional version is denoted by $\mathcal{S}_{n}^{\mathcal{E}}$.

The pairing between a matrix space and its conjugate arises from the parallel duality: for (finite or infinite) matrices $T=\left(t_{i j}\right)$ and $S=\left(s_{i j}\right),\langle T, S\rangle=\operatorname{Tr} T^{t} S=$ $\sum_{i, j} t_{i j} s_{i j}\left({ }^{t} S\right.$ is the transpose of $S$ ).

For a matrix $\phi=\left(\phi_{i j}\right)_{i, j \in I}$, we define a Schur multiplier $\mathbb{S}_{\phi}$ : for any matrix $T=\left(t_{i j}\right) \in \mathfrak{X}$ (where $\mathfrak{X}$ is an unconditional matrix space), $\mathbb{S}_{\phi} T=\phi \diamond T=\left(\phi_{i j} t_{i j}\right)$. The matrix $\phi$ is called the symbol of the Schur multiplier. If $\mathbb{S}_{\phi}$ is viewed as an operator from $\mathfrak{X}$ to $\mathfrak{Y}$, we use the notation $\mathbb{S}_{\phi}^{\mathfrak{X}, \mathfrak{Y}}$. If $\mathfrak{X}=\mathfrak{Y}$, we write $\mathbb{S}_{\phi}^{\mathfrak{X}}$. If $\mathfrak{X}=\mathcal{S}^{\mathcal{E}}$ and $\mathfrak{Y}=\mathcal{S}^{\mathcal{F}}$, we write $\mathbb{S}_{\phi}^{\mathcal{E}, \mathcal{F}}$. Furthermore, if $\mathcal{E}=\ell_{p}$ and $\mathcal{F}=\ell_{q}$, we write $\mathbb{S}_{\phi}^{p, q}$.

For $\mathbf{S} \subset I \times I$, denote by $\mathbb{S}_{\phi, \mathbf{S}}$ the restriction of $\mathbb{S}_{\phi}$ to $\operatorname{span}\left[E_{i j}:(i, j) \in \mathbf{S}\right]$. Here, $\left(E_{i j}\right)_{i, j \in I}$ are matrix units, that is, the matrices with 1 in the $(i, j)$ position and zeroes elsewhere. To denote restricted Schur multipliers between specific spaces, we use the notation $\mathbb{S}_{\phi, \mathbf{X}}^{\mathfrak{X}, \mathfrak{Y}}$, etc. We denote by $\mathfrak{X}_{\mathbf{S}}$ the set of all $T=\left(t_{i j}\right) \in \mathfrak{X}$ such that $t_{i j}=0$ when $(i, j) \notin \mathbf{S}$.

Throughout this paper, we are primarily concerned with elementary Schur multipliers - that is, those for which the symbol $\phi$ satisfies $\phi_{i j}=a_{i} b_{j}$, for sequences $a=\left(a_{i}\right), b=\left(b_{j}\right) \in \ell_{\infty}$. In this case, we write $\phi=a \cdot b$.

Theorem 1.1. Suppose $\mathfrak{X}$ is an unconditional matrix space, $\mathbf{S}$ is a subset of $\mathbb{N} \times \mathbb{N}$, and $a=\left(a_{i}\right), b=\left(b_{j}\right)$ are sequences of scalars. Then $\left\|\mathbb{S}_{a \cdot b, \mathbf{S}}^{\mathfrak{X}}\right\|=\sup _{(i, j) \in \mathbf{S}}\left|a_{i} b_{j}\right|$.

Note that, in particular, $\left\|\mathbb{S}_{a \cdot b}\right\|=\sup _{i}\left|a_{i}\right| \sup _{j}\left|b_{j}\right|$; in fact, this is evident from the unconditionality of our matrix spaces. In general, computing, or even estimating, the norms of Schur multipliers - to say nothing of their restrictions - is extremely tough. Perhaps the best explored case is that of multipliers on $B\left(\ell_{2}\right)$. There, the importance of elementary multipliers is apparent: by Grothendieck's Theorem, a Schur multiplier is bounded iff it lies in the pointwise closure of the convex hull of the elementary ones (see e.g. Chapter 5 of [25]). Surprisingly, the norm of a "random" Schur multiplier is much easier to handle 24. The same techniques were further refined in 7 . That paper characterizes the matrices $A$ with the property that any " $A$-dominated" Schur multiplier is bounded. Compactness of Schur multipliers on $B\left(\ell_{2}\right)$ was described in [15].

Much less is known about Schur multipliers on the Schatten spaces $\mathcal{S}^{p}$. It was shown in 13 that, for any even integer $p$ and any $q>p$, there exists a matrix $\phi$ s.t. $\mathbb{S}_{\phi}$ is bounded on $\mathcal{S}^{p}$ but not on $\mathcal{S}^{q}$. The boundedness of various Schur multipliers was established in [10, 14], while [4] contains lower estimates on the norms of Schur idempotents. A special class of Schur multipliers was recently investigated in 27. The norm estimates obtained there were used to show that Lipschitz functions operate continuously on self-adjoint elements of $\mathcal{S}^{p}(1<p<\infty)$.

Theorem 1.1 yields a generalization of the Wielandt minimax principle.

Theorem 1.2. Suppose $\mathcal{E}$ is a symmetric sequence space, $H_{1}$ and $H_{2}$ are Hilbert spaces, and $A \in K\left(H_{1}, H_{2}\right)$ has singular values $a_{1} \geqslant a_{2} \geqslant \ldots \geqslant 0$. Suppose, furthermore, that $n \in \mathbb{N}$ and $i_{1}<i_{2}<\ldots<i_{n}$ are positive integers. Then:

$$
\left\|\left(a_{i_{1}}, \ldots, a_{i_{n}}\right)\right\|_{\mathcal{E}}=\sup _{\left(E_{k}\right)} \inf _{\left(e_{k}\right)}\left\|\left.A\right|_{\operatorname{span}\left[e_{1}, \ldots, e_{n}\right]}\right\|_{\mathcal{E}}
$$


Here, $\sup _{\left(E_{k}\right)}$ is taken over all families of subspaces of $E$ with $E_{1} \hookrightarrow E_{2} \hookrightarrow$ $\ldots \hookrightarrow E_{n}$ and $\operatorname{dim} E_{k}=i_{k}$, while $\inf _{\left(e_{k}\right)}$ is taken over all orthonormal systems $\left(e_{k}\right)_{k=1}^{n}$, with $e_{k} \in E_{k}$ for every $k$.

(2)

$$
\left\|\left(a_{i_{1}}, \ldots, a_{i_{n}}\right)\right\|_{\mathcal{E}}=\inf _{\left(E_{k}\right)} \sup _{\left(e_{k}\right)}\left\|\left.A\right|_{\operatorname{span}\left[e_{1}, \ldots, e_{n}\right]}\right\|_{\mathcal{E}} .
$$

Here, $\inf _{\left(E_{k}\right)}$ is taken over all families of subspaces of $E$ with $E_{n} \hookrightarrow$ $E_{n-1} \hookrightarrow \ldots \hookrightarrow E_{1}$ and codim $E_{k}=i_{k}-1$, while $\sup _{\left(e_{k}\right)}$ is taken over all orthonormal systems $\left(e_{k}\right)_{k=1}^{n}$, with $e_{k} \in E_{k}$ for every $k$.

If $\mathcal{E}$ is 2-convex (for instance, if $\mathcal{E}=\ell_{p}$, with $p \geqslant 2$ ), the above result follows from Wielandt-style inequalities on eigenvalues (see e.g. Section III.3 of [5]).

Theorem 1.1 also allows us to compute various $s$-numbers of elementary Schur multipliers on $B\left(\ell_{2}\right)$. We refer the reader to [23] for general information about $s$-numbers, and to [17] (and references therein) for their non-commutative versions. General theory concerning operator spaces can be found in [11, 18, 26]. Below, we recall some necessary definitions.

Suppose $T$ is a linear operator between Banach (or operator) spaces $X$ and $Y$. We define the approximation numbers $a_{n}$, Kolmogorov numbers $d_{n}$, and Gelfand numbers $c_{n}$ as follows:

$$
\begin{aligned}
& a_{n}(T)=\inf \{\|T-S\|: S \in B(X, Y), \text { rank } S<n\} \\
& d_{n}(T)=\inf \{\|q T\|: q: Y \rightarrow Y / F \text { is a quotient map, } \operatorname{dim} F<n\} \\
& c_{n}(T)=\inf \left\{\left\|\left.T\right|_{E}\right\|: E \hookrightarrow X, \operatorname{codim} E<n\right\} .
\end{aligned}
$$

The non-commutative versions of these sequences are defined similarly, with $a_{n}^{(c b)}(T)$ $=\inf \left\{\|T-S\|_{c b}: S \in B(X, Y)\right.$, rank $\left.S<n\right\}$, etc.

It is known that $\lim _{n} d_{n}(T)=0$ iff $\lim _{n} c_{n}(T)=0$ iff $T$ is compact. For this reason, we are mostly interested in non-zero compact elementary Schur multipliers. It is easy to see that $\mathbb{S}_{a \cdot b} \neq 0$ is compact iff $a, b \in c_{0}$. In this case, denote by $\widetilde{[a b]}$ the non-increasing rearrangement of the double sequence $\left(a_{i} b_{j}\right)$.

Theorem 1.3. For $a, b \in c_{0}$, consider $\mathbb{S}=\mathbb{S}_{a \cdot b}$ as an operator on an unconditional matrix space $\mathfrak{X}$. Then $a_{n}(\mathbb{S})=c_{n}(\mathbb{S})=d_{n}(\mathbb{S})=\widetilde{[a b}_{n}$ for any $n \in \mathbb{N}$. If $\mathbb{S}$ acts on $B\left(\ell_{2}\right)$ or $\mathcal{S}^{p}(1 \leqslant p \leqslant \infty)$, then, in addition, $a_{n}^{(c b)}(\mathbb{S})=c_{n}^{(c b)}(\mathbb{S})=d_{n}^{(c b)}(\mathbb{S})=\widetilde{[a b}_{n}$.

The information about the behavior of $s$-numbers of an elementary Schur multiplier can be used to determine its membership in various operator ideals (see e.g. 22, 23] for the definitions and basic properties). Some results of this type are contained in Section 5 .

We prove Theorem 1.1 in Section 2, Section 3 is devoted to establishing Theorem 1.2. The proof of Theorem 1.3 is given in Section 4.

\section{Proof of Theorem 1.1}

Suppose $\mathfrak{X}$ is an unconditional matrix space on $I \times I$. For $J_{1}, J_{2} \subset I$, define the truncation map $\mathcal{P}_{J_{1}, J_{2}}$ by setting, for $\left(a_{i j}\right) \in \mathfrak{X}$,

$$
\left(\mathcal{P}_{J_{1}, J_{2}}\left(a_{i j}\right)\right)_{p q}=\left\{\begin{array}{ll}
a_{p q} & (p, q) \in J_{1} \times J_{2} \\
0 & (p, q) \notin J_{1} \times J_{2}
\end{array} .\right.
$$


Lemma 2.1. Suppose $\mathfrak{X}$ is an unconditional matrix space on $I \times I, J_{1}, J_{2} \subset I$, and $T \in \mathfrak{X}$ satisfies $\mathcal{P}_{J_{1}^{c}, J_{2}^{c}} T=0$. Then, for $\lambda \in(0,1), \| \mathcal{P}_{J_{1}, J_{2}^{c}} T+\mathcal{P}_{J_{1}^{c}, J_{2}} T+$ $\lambda \mathcal{P}_{J_{1}, J_{2}} T\left\|_{\mathfrak{X}} \leqslant\right\| T \|_{\mathfrak{X}}$.

Proof. Write $T=\left(t_{i j}\right)$, and let

$$
T_{1}=\mathcal{P}_{J_{1}, J_{2}^{c}} T+\mathcal{P}_{J_{1}^{c}, J_{2}} T=\frac{T+T_{0}}{2}, \text { where }\left(T_{0}\right)_{i j}=(-1)^{\chi_{J_{1}}(i)}(-1)^{\chi_{J_{2}}(j)} t_{i j} .
$$

As $\mathfrak{X}$ is an unconditional matrix space, $\left\|T_{1}\right\| \leqslant\|T\|=\left\|T_{0}\right\|$. Note that $T=$ $T_{1}+P T Q$, hence

$$
\left\|T_{1}+\lambda P T Q\right\|_{\mathfrak{X}}=\left\|\lambda T+(1-\lambda) T_{1}\right\|_{\mathfrak{X}} \leqslant\|T\|_{\mathfrak{X}}=1,
$$

by the triangle inequality.

Proof of Theorem 1.1. One direction is clear:

$$
\left\|\mathbb{S}_{a \cdot b, \mathbf{S}}^{\mathfrak{X}}\right\| \geqslant \sup _{(i, j) \in \mathbf{S}}\left\|\mathbb{S}_{a \cdot b, \mathbf{S}}^{\mathfrak{X}} E_{i j}\right\|=\sup _{(i, j) \in \mathbf{S}}\left|a_{i} b_{j}\right| .
$$

To establish the opposite inequality, we can assume that $\mathbf{S}$ is a subset of $\{1, \ldots, N\} \times$ $\{1, \ldots, N\}$, and that $a_{1} \geqslant a_{2} \geqslant \ldots \geqslant a_{N}>0$ and $b_{1} \geqslant b_{2} \geqslant \ldots \geqslant b_{N}>0$. By re-scaling and enlarging the set $\mathbf{S}$ if necessary, we can assume that $\mathbf{S}=\{(i, j)$ : $\left.a_{i} b_{j} \leqslant 1\right\}$. It suffices to show that, for such $\mathbf{S}$,

$$
\left\|\mathbb{S}_{a \cdot b, \mathbf{S}}^{\mathfrak{X}}\right\| \leqslant 1 \text {. }
$$

By increasing $N$ and extending the sequences $a$ and $b$, we can assume that the points $(1, N)$ and $(N, 1)$ belong to $\mathbf{S}$. Clearly, there exists $n<N$ and sequences $1 \leqslant i_{1}<i_{2}<\ldots<i_{n}<N$ and $1 \leqslant j_{1}<j_{2}<\ldots<j_{n}<N$ such that $\mathbf{S}^{c}=$ $\bigcup_{s=1}^{n}\left[1, i_{s}\right] \times\left[1, j_{n+1-s}\right]$. Moreover, such sequences (and $n=n(\mathbf{S})$ ) are uniquely determined by the set $\mathbf{S}$. For notational simplicity, let $i_{0}=j_{0}=0$ and $i_{n+1}=$ $j_{n+1}=N$.

Note that $\mathbb{S}_{a^{\prime} a^{\prime \prime} \cdot b^{\prime \prime} b^{\prime}}=\mathbb{S}_{a^{\prime} \cdot b^{\prime}} \mathbb{S}_{a^{\prime \prime} \cdot b^{\prime \prime}}$ (the product of sequences $a^{\prime}$ and $a^{\prime \prime}$ is taken to be the pointwise one) and that $\mathbb{S}_{a^{\prime} \cdot b^{\prime}}$ is a contraction whenever $a^{\prime}$ and $b^{\prime}$ belong to the unit ball of $\ell_{\infty}$. Therefore, by increasing (some of) the $a_{i}$ 's and $b_{j}$ 's, we can assume that $a_{k}=a_{i_{s}+1}=\alpha_{s}$ when $i_{s}<k \leqslant i_{s+1}$ and $b_{k}=b_{j_{s}+1}=\beta_{s}$ when $j_{s}<k \leqslant j_{s+1}$. Furthermore, we can assume that $\alpha_{s} \beta_{n-s}=1$ for $0 \leqslant s \leqslant n$. We are going to establish (2.1) under these assumptions by using induction on $n=n(\mathbf{S})$.

The case of $n=0$ is trivial: here $\mathbf{S}^{c}=\emptyset$; hence $\left|a_{1} b_{1}\right| \leqslant 1$ and $\mathbb{S}_{a \cdot b}$ is contractive. Next consider the case of $n=1$. Then $\mathbf{S}^{c}=\left[1, i_{1}\right] \times\left[1, j_{1}\right], a_{k}=\alpha_{0}$ for $i_{0}+1=1 \leqslant$ $k \leqslant i_{1}, a_{k}=\alpha_{1}$ for $i_{1}+1 \leqslant k \leqslant i_{2}=N, b_{k}=\beta_{0}=1 / \alpha_{1}$ for $j_{0}+1=1 \leqslant k \leqslant j_{1}$, and $b_{k}=\beta_{1}=1 / \alpha_{0}$ for $j_{1}+1 \leqslant k \leqslant j_{2}=N$. Suppose $T=\sum_{(i, j) \in \mathbf{S}} t_{i j} E_{i j}$ satisfies $\|T\|_{\mathfrak{X}} \leqslant 1$; we show that $\left\|\mathbb{S}_{a \cdot b} T\right\|_{\mathfrak{X}} \leqslant 1$. Indeed, write $T=T_{1}+T_{2}$, where

$$
T_{1}=\sum_{(i, j) \in\left[1, i_{1}\right] \times\left[j_{1}+1, N\right] \cup\left[i_{1}+1, N\right] \times\left[1, j_{1}\right]} t_{i j} E_{i j} \text { and } T_{2}=\sum_{(i, j) \in\left[i_{1}+1, N\right] \times\left[j_{1}+1, N\right]} t_{i j} E_{i j} .
$$

In other words, $T_{1}=\mathcal{P}_{J_{1}, J_{2}^{c}} T+\mathcal{P}_{J_{1}^{c}, J_{2}} T$ and $T_{2}=\mathcal{P}_{J_{1}, J_{2}} T$, where $J_{1}=\left\{k: k>i_{1}\right\}$ and $J_{2}=\left\{k: k>j_{1}\right\}$. Note that $\mathcal{P}_{J_{1}^{c}, J_{2}^{c}} T=0$. Then $\mathbb{S}_{a \cdot b} T=T_{1}+\lambda T_{2}$, with $\lambda=\alpha_{1} / \alpha_{0} \in(0,1)$. By Lemma 2.1, $\left\|\mathbb{S}_{a \cdot b} T\right\|_{\mathfrak{X}} \leqslant 1$.

Now consider general $n>1$. As before, we are assuming that $a_{k}=\alpha_{s}$ for $i_{s}<k \leqslant i_{s+1}$ and $b_{k}=\beta_{s}=1 / \alpha_{n-s}$ for $j_{s}<k \leqslant j_{s+1}$. Modify these sequences by 
letting

$$
a_{k}^{\prime}=\left\{\begin{array}{ll}
a_{k} & 1 \leqslant k \leqslant i_{n} \\
\alpha_{n-1} & i_{n}+1 \leqslant k \leqslant N
\end{array} \quad, \quad b_{k}^{\prime}=\left\{\begin{array}{ll}
b_{k} & j_{1}+1 \leqslant k \leqslant N \\
\beta_{1} & 1 \leqslant k \leqslant j_{1}
\end{array} .\right.\right.
$$

Note that $a_{k}^{\prime}=\alpha_{n-1}$ iff $i_{n-1}<k \leqslant i_{n+1}=N, b_{k}^{\prime}=\beta_{1}$ iff $0=i_{0}<k \leqslant j_{2}$, and

$$
a_{k}^{\prime} b_{\ell}^{\prime} \leqslant 1 \text { iff }(k, \ell) \in \mathbf{S}^{\prime}=\left(\bigcup_{s=1}^{n-1}\left[1, i_{s}\right] \times\left[1, j_{n+1-s}\right]\right)^{c} \supset \mathbf{S} .
$$

Thus, $n\left(\mathbf{S}^{\prime}\right)=n-1$ and hence, by the induction hypothesis, $\mathbb{S}_{a^{\prime} \cdot b^{\prime}}$ determines a contraction on $\mathfrak{X}_{\mathbf{S}^{\prime}}$. Furthermore, for $(k, \ell) \in \mathbf{S}$,

$$
\frac{a_{k} b_{\ell}}{a_{k}^{\prime} b_{\ell}^{\prime}}=\left\{\begin{array}{ll}
1 & (k, \ell) \notin\left[i_{n}+1, N\right] \times\left[j_{1}+1, N\right] \\
\lambda=\alpha_{n} / \alpha_{n-1} & (k, \ell) \in\left[i_{n}+1, N\right] \times\left[j_{1}+1, N\right]
\end{array} .\right.
$$

Suppose $T=\left(t_{i j}\right)_{(i, j) \in \mathbf{S}}$ satisfies $\|T\|_{\mathfrak{X}} \leqslant 1$; we show that $\left\|\mathbb{S}_{a \cdot b} T\right\|_{\mathfrak{X}} \leqslant 1$. Let $T^{\prime}=\mathbb{S}_{a^{\prime} \cdot b^{\prime}} T$ (i.e. $T^{\prime}=\left(t_{i j}^{\prime}\right)$, with $t_{i j}^{\prime}=a_{i}^{\prime} b_{j}^{\prime} t_{i j}$ ). By the induction hypothesis, $\left\|T^{\prime}\right\|_{\mathfrak{X}} \leqslant 1$. We have to show that $\left\|\mathbb{S}_{a \cdot b} T\right\|_{\mathfrak{X}} \leqslant\left\|T^{\prime}\right\|_{\mathfrak{X}}$. To this end, write $T^{\prime}=$ $T_{1}+T_{2}$, where

$$
T_{1}=\sum_{(i, j) \in\left[1, i_{n}\right] \times\left[j_{1}+1, N\right] \cup\left[i_{n}+1, N\right] \times\left[1, j_{1}\right]} t_{i j}^{\prime} E_{i j} \text { and } T_{2}=\sum_{(i, j) \in\left[i_{n}+1, N\right] \times\left[j_{1}+1, N\right]} t_{i j}^{\prime} E_{i j} .
$$

In other words, $T_{1}=\mathcal{P}_{J_{1}, J_{2}^{c}} T+\mathcal{P}_{J_{1}^{c}, J_{2}} T$ and $T_{2}=\mathcal{P}_{J_{1}, J_{2}} T$, where $J_{1}=\left\{k: k>i_{n}\right\}$ and $J_{2}=\left\{k: k>j_{1}\right\}$. By (2.2), $\mathbb{S}_{a \cdot b} T=T_{1}+\lambda T_{2}$. Thus, by Lemma 2.1] $\left\|\mathbb{S}_{a, b} T\right\|_{\mathfrak{X}} \leqslant\left\|T^{\prime}\right\|_{\mathfrak{X}} \leqslant\|T\|_{\mathfrak{X}}$. This proves (2.1) and, therefore, Theorem 1.1.

Corollary 2.2. Suppose $\mathfrak{X}$ is an unconditional matrix space on $I \times I, \mathbf{S}$ is a subset of $I \times I$, and $\left(a_{i}\right),\left(b_{j}\right)$ are sequences of scalars. Then, for any $x \in \mathfrak{X}_{\mathbf{S}}$,

$$
\left\|\mathbb{S}_{a \cdot b} x\right\|_{\mathfrak{X}} \geqslant \inf _{(i, j) \in \mathbf{S}}\left|a_{i} b_{j}\right|\|x\|_{\mathfrak{X}} .
$$

Proof. We can assume that the set $\mathbf{S}$ is finite and $\inf _{(i, j) \in \mathbf{S}}\left|a_{i} b_{j}\right|=c>0$. By truncating, we can assume that the operator $\mathbb{S}_{a \cdot b}$ acts on the space of $N \times N$ matrices, for some $N$, and that $a_{1} \geqslant a_{2} \geqslant \ldots \geqslant a_{N}>0$ and $b_{1} \geqslant b_{2} \geqslant \ldots \geqslant b_{N}>0$. Consider the operator $\mathbb{S}_{a^{\prime} \cdot b^{\prime}}$, where $a_{i}^{\prime}=1 / a_{i}$ and $b_{j}^{\prime}=1 / b_{j}$. By Theorem 1.1, $\left\|\mathbb{S}_{a^{\prime} \cdot b^{\prime}, \mathbf{S}}^{\mathfrak{X}}\right\|=\sup _{(i, j) \in \mathbf{S}} a_{i}^{\prime} b_{j}^{\prime}=1 / c$. As $\mathbb{S}_{a^{\prime} \cdot b^{\prime}} \mathbb{S}_{a \cdot b}=I$, we are done.

\section{Proof of Theorem 1.2}

Proof. Without loss of generality, assume that $H_{1}=H_{2}=H$ and that the operator $A$ is positive and diagonal. By a small perturbation argument, we can assume that $H=\ell_{2}^{N}$ for some $N \geqslant i_{n}$ and $A \delta_{s}=a_{s} \delta_{s}$ for $1 \leqslant s \leqslant N\left(\left(\delta_{s}\right)_{s=1}^{N}\right.$ stands for the canonical basis for $\left.\ell_{2}^{N}\right)$. Furthermore, assume that $a_{1} \geqslant a_{2} \geqslant \ldots \geqslant a_{n}>0$.

(1) By taking $e_{k}=\delta_{i_{k}}$, we obtain

$$
\left\|\left(a_{i_{1}}, \ldots, a_{i_{n}}\right)\right\|_{\mathcal{E}} \leqslant \sup _{\left(E_{k}\right)} \inf _{\left(e_{k}\right)}\left\|\left.A\right|_{\operatorname{span}\left[e_{1}, \ldots, e_{n}\right]}\right\|_{\mathcal{E} .} .
$$

To prove the opposite inequality, suppose $E_{1} \hookrightarrow \ldots \hookrightarrow E_{n}$ and $e_{k} \in E_{k}$ are as in the statement of the theorem. Let $F_{k}=\operatorname{span}\left[\delta_{s}: s \geqslant i_{k}\right]$. By Theorem III.3.2 and Exercise III.3.3 of [5], we can find an orthonormal system $\left(f_{k}\right)$ s.t. $f_{k} \in F_{k}$ for each $k$ and $\operatorname{span}\left[e_{1}, \ldots, e_{n}\right]=\operatorname{span}\left[f_{1}, \ldots, f_{n}\right]$. Denote this space by $F$. It remains to show that

$$
\left\|\left.A\right|_{F}\right\|_{\mathcal{E}} \leqslant\left\|\left(a_{i_{1}}, \ldots, a_{i_{n}}\right)\right\|_{\mathcal{E}}=\left\|\left.A\right|_{E}\right\|_{\mathcal{E}}
$$


where $E=\operatorname{span}\left[\delta_{i_{1}}, \ldots, \delta_{i_{n}}\right]$. To this end, define an isometry $U: F \rightarrow E: f_{k} \mapsto \delta_{i_{k}}$ and an operator $T: E \rightarrow A(F): \delta_{i_{k}} \mapsto A f_{k} / a_{i_{k}}$. Then $\left.A\right|_{F}=\left.T \circ A\right|_{E} \circ U$. By the ideal property of $\|\cdot\|_{\mathcal{E}}$, it suffices to show that $\|T\| \leqslant 1$.

Write $f_{k}=\sum_{j=i_{k}}^{N} \gamma_{k j} \delta_{j}$. Then $T \delta_{i_{k}}=\sum_{j=i_{k}}^{N} \gamma_{k j} a_{j} a_{i_{k}}^{-1} \delta_{j}$. But $a_{j} \leqslant a_{i_{k}}^{-1}$ whenever $\gamma_{k j} \neq 0$; hence, by Theorem 1.1.

$$
\left\|\left(\gamma_{k j} a_{j} a_{i_{k}}^{-1}\right)_{1 \leqslant k \leqslant n, 1 \leqslant j \leqslant N}\right\| \leqslant\left\|\left(\gamma_{k j}\right)_{1 \leqslant k \leqslant n, 1 \leqslant j \leqslant N}\right\|=1
$$

(the latter is true, since the rows of the matrix $\left(\gamma_{k j}\right)$ are orthonormal vectors). This establishes (3.1), thus completing the proof of part (1) of the theorem.

(2) is established in a similar fashion, so we only sketch the proof. The inequality

$$
\left\|\left(a_{i_{1}}, \ldots, a_{i_{n}}\right)\right\|_{\mathcal{E}} \geqslant \inf _{\left(E_{k}\right)} \sup _{\left(e_{k}\right)}\left\|\left.A\right|_{\operatorname{span}\left[e_{1}, \ldots, e_{n}\right]}\right\|_{\mathcal{E}}
$$

is obvious. To establish the converse, consider $\left(E_{k}\right)$ and $\left(e_{k}\right)$ as in the statement of the theorem. Let $F=\operatorname{span}\left[e_{1}, \ldots, e_{n}\right]$ and $F_{k}=\operatorname{span}\left[\delta_{s}: s \leqslant i_{k}\right](1 \leqslant k \leqslant n)$. As in part (1), we show the existence of an orthonormal system $\left(f_{k}\right)$ s.t. $f_{k} \in F_{k}$ for each $k$ and $F=\operatorname{span}\left[f_{1}, \ldots, f_{n}\right]$. To show that $\left\|\left.A\right|_{F}\right\|_{\mathcal{E}} \geqslant\left\|\left(a_{i_{1}}, \ldots, a_{i_{n}}\right)\right\|_{\mathcal{E}}$, we proceed as in part (1), except that now we have to show that $T: \delta_{i_{k}} \mapsto A f_{k} / a_{i_{k}}$ has a contractive inverse. To this end, use Corollary 2.2.

Remark 3.1. Alternatively, one can deduce part (1) from Theorem 2.15 of [1].

\section{Proof of Theorem 1.3}

We can assume that $I=\mathbb{N}, a_{1} \geqslant a_{2} \geqslant \ldots \geqslant 0$, and $b_{1} \geqslant b_{2} \geqslant \ldots \geqslant 0$. For notational simplicity, we let $\lambda=\widetilde{[a b]}$ - that is, $\lambda_{m}$ is the $m$-th largest element of $\left(a_{i} b_{j}\right)_{i, j \in \mathbb{N}}$. Denote $\mathbb{S}_{a \cdot b}$ (acting on $\mathfrak{X}$ ) simply by $\mathbb{S}$.

Recall two little known $s$-sequences (see [20]): for $T \in B(X, Y)$, define the $n$-th Bernstein number by

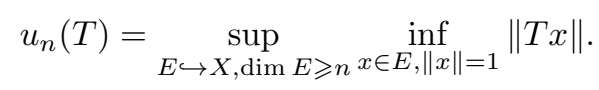

The $n$-th Mitiagin number $v_{n}(T)$ is defined as

$$
v_{n}(T)=\sup _{F \hookrightarrow Y, \operatorname{codim} F \geqslant n} \rho\left(q_{F} T\right),
$$

where $q_{F}: Y \rightarrow Y / F$ is the quotient map and, for an operator $S: Z \mapsto W$,

$$
\rho(S)=\sup \left\{c \geqslant 0: c \mathbf{B}_{W} \subset S\left(\mathbf{B}_{Z}\right)\right\}
$$

( $\mathbf{B}_{W}$ and $\mathbf{B}_{Z}$ refer to the unit balls of $W$ and $Z$, respectively).

Lemma 4.1. With the above notation, suppose $\mathbb{S}$ is acting on an unconditional matrix space $\mathfrak{X}$. Then, for every $m \in \mathbb{N}, \lambda_{m} \leqslant \min \left\{u_{m}(\mathbb{S}), v_{m}(\mathbb{S})\right\}$.

Proof. To prove $\lambda_{m} \leqslant u_{m}(\mathbb{S})$, let $\mathbf{S}=\left\{(i, j): a_{i} b_{j}<\lambda_{m}\right\}$ and $E=\operatorname{span}\left[E_{i j}\right.$ : $(i, j) \notin \mathbf{S}]$. Then $\operatorname{dim} E \geqslant m$ and, by Corollary 2.2. $\|\mathbb{S} x\| \geqslant \lambda_{m}\|x\|$ for any $x \in E$.

The inequality $\lambda_{m} \leqslant v_{m}(\mathbb{S})$ follows by duality. Indeed, denote by $\mathcal{P}_{N}$ the operator of truncation to the top left $N \times N$ corner, and let $\mathfrak{X}_{N}$ be the range of $\mathcal{P}_{N}$ in $\mathfrak{X}$. Both $\mathfrak{X}_{N}$ and its dual $\mathfrak{X}_{N}^{*}$ are unconditional matrix spaces. The previous paragraph shows that, for $N$ sufficiently large, $u_{m}\left(\mathbb{S}_{a \cdot b}^{\mathfrak{X}} \mathcal{P}_{N}\right) \geqslant \lambda_{m}$, where $\mathbb{S}_{a \cdot b}^{\mathfrak{X}} \mathcal{P}_{N}$ is viewed as an operator on $\mathfrak{X}_{N}$. By [20] (Lemma 6.1 and Theorem 6.4), $v_{m}(T)=u_{m}\left(T^{*}\right)$ for 
any linear operator $T$. Therefore,

$$
v_{m}(\mathbb{S}) \geqslant v_{m}\left(\mathbb{S P}_{N}\right)=u_{m}\left(\left(\mathbb{S P}_{N}\right)^{*}\right)=u_{m}\left(\mathbb{S}^{*} \mathcal{P}_{N}\right) \geqslant \lambda_{m}
$$

(here, we identify $\mathbb{S}^{*} \mathcal{P}_{N}$ with the operator $\mathbb{S}_{\mathcal{P}}$ acting on $\mathfrak{X}_{N}^{*}$ ).

Lemma 4.2. With the above notation, $\lambda_{m} \geqslant a_{m}(\mathbb{S})$. If, in addition, $\mathbb{S}$ acts on $B\left(\ell_{2}\right)$ or $\mathcal{S}^{p}(1 \leqslant p \leqslant \infty)$, then $\lambda_{m} \geqslant a_{m}^{(c b)}(\mathbb{S})$.

Proof. Let $\mathbf{S}=\left\{(i, j): a_{i} b_{j} \leqslant \lambda_{m}\right\}$. Then $\left|\mathbf{S}^{c}\right|<m$, and $\left\|\left.\mathbb{S}\right|_{\mathbf{S}}\right\| \leqslant \lambda_{m}$. By scaling, we can assume that $\lambda_{n}=1$. As in the proof of Theorem [1.1, we can assume that there exist $n \in \mathbb{N}, 0=i_{0}<i_{1}<\ldots<i_{n}<i_{n+1}=\infty$, and $0=j_{0}<j_{1}<\ldots<$ $j_{n}<j_{n+1}=\infty$ such that $a_{k}=a_{i_{s}+1}=\alpha_{s}$ when $i_{s}<k \leqslant i_{s+1}$ and $b_{k}=b_{j_{s}+1}=\beta_{s}$ when $j_{s}<k \leqslant j_{s+1}$. Furthermore, we can assume that $\alpha_{s} \beta_{n-s}=1$ for $0 \leqslant s \leqslant n$ and $\alpha_{0}>\alpha_{1}>\ldots>\alpha_{n}$.

For $1 \leqslant s \leqslant n$, let $\gamma_{s}=\alpha_{s} / \alpha_{s-1}$, and consider the sequences

$$
c^{(s)}=(-1)^{\chi_{\left\{1, \ldots, i_{s}\right\}}} \text { and } d^{(s)}=(-1)^{\chi_{\left\{j_{n-s+1}+1, j_{n-s+1}+2, \ldots\right\}}} \text {. }
$$

In other words,

$$
c_{k}^{(s)}=\left\{\begin{array}{ll}
-1 & k \leqslant i_{s} \\
1 & k>i_{s}
\end{array} \text { and } d_{\ell}^{(s)}=\left\{\begin{array}{ll}
1 & \ell \leqslant j_{n-s+1} \\
-1 & \ell>j_{n-s+1}
\end{array} .\right.\right.
$$

Define the Schur multipliers

$$
\mathbb{S}_{\phi^{(s)}}=\frac{1+\gamma_{s}}{2}+\frac{1-\gamma_{s}}{2} \mathbb{S}_{c^{(s)} \cdot d^{(s)}}
$$

Note that the symbol $\phi^{(s)}$ of this multiplier satisfies

$$
\phi_{k \ell}^{(s)}=\left\{\begin{array}{ll}
1 & (k, \ell) \in\left[1, i_{s}\right] \times\left(j_{n-s+1}, \infty\right) \cup\left(i_{s}, \infty\right) \times\left[1, j_{n-s+1}\right] \\
\gamma_{s} & (k, \ell) \in\left[1, i_{s}\right] \times\left[1, j_{n-s+1}\right] \cup\left(i_{s}, \infty\right) \times\left(j_{n-s+1}, \infty\right)
\end{array} .\right.
$$

Let $\phi=\phi^{(1)} \diamond \ldots \diamond \phi^{(n)}$. By (4.1) $\mathbb{S}_{\phi}=\mathbb{S}_{\phi^{(1)}} \ldots \mathbb{S}_{\phi^{(n)}}$ is a contraction.

Next we show that, for $(k, \ell) \in \mathbf{S}$,

$$
\phi_{k \ell}=a_{k} b_{\ell} .
$$

To this end, fix $(k, \ell)$, and find $p, q \in\{0, \ldots, n\}$ s.t. $p+q \geqslant n, i_{p}<k \leqslant i_{p+1}$, and $j_{q}<\ell \leqslant j_{q+1}$. Then $a_{k} b_{\ell}=\alpha_{p} \beta_{q}$.

First suppose $p+q=n(q=n-p)$. Then, for every $s$,

$$
(k, \ell) \in\left(i_{p}, i_{p+1}\right] \times\left(j_{n-p}, j_{n-p+1}\right] \subset\left[1, i_{s}\right] \times\left(j_{n-s+1}, \infty\right) \cup\left(i_{s}, \infty\right) \times\left[1, j_{n-s+1}\right]
$$

(to see this, consider the cases of $s \leqslant p+1$ and $s \geqslant p$ separately). In this case, $a_{k} b_{\ell}=1$ and, by (4.2), $\phi_{k \ell}^{(s)}=1$ for every $s$; hence $\phi_{k \ell}=1$.

Now suppose $q>n-p$. As $\gamma_{s}=\alpha_{s-1} / \alpha_{s}$, we have

$$
a_{k} b_{\ell}=\alpha_{p} \beta_{q}=\frac{\alpha_{p}}{\alpha_{n-q}}=\prod_{s=n-q+1}^{p} \gamma_{s} .
$$

On the other hand, by (4.2), $\phi_{k \ell}^{(s)}=\gamma_{s}$ iff $n-q+1 \leqslant s \leqslant p$; hence $\phi_{k \ell}=$ $\gamma_{n-q+1} \ldots \gamma_{p}$, thus establishing (4.3).

Now let $\psi=a \cdot b-\phi$. Then $\psi$ has fewer than $m$ non-zero entries, and $\mathbb{S}_{a \cdot b}-\mathbb{S}_{\psi}=$ $\mathbb{S}_{\phi}$. Therefore,

as desired.

$$
a_{m}\left(\mathbb{S}_{a \cdot b}\right) \leqslant\left\|\mathbb{S}_{\psi}\right\|=1=\lambda_{m}
$$


It remains to estimate $\left\|\mathbb{S}_{\phi}\right\|_{c b}$ when $\mathfrak{X}$ is $B\left(\ell_{2}\right)$ or $\mathcal{S}^{p}$, for $1 \leqslant p \leqslant \infty$. It is well known that the norm and c.b. norm of any Schur multiplier on $B\left(\ell_{2}\right)$ coincide. By [15], $\mathbb{S}_{\phi}$ maps $\mathcal{S}^{\infty}$ into itself; hence, by "parallel duality," it maps $\mathcal{S}^{1}$ into itself, with

$$
\left\|\mathbb{S}_{\phi}\right\|_{C B\left(\mathcal{S}^{1}\right)}=\left\|\mathbb{S}_{\phi}\right\|_{C B\left(\mathcal{S}^{\infty}\right)}=\left\|\mathbb{S}_{\phi}\right\|_{B\left(B\left(\ell_{2}\right)\right)}=1 .
$$

Complex interpolation yields $\left\|\mathbb{S}_{\phi}\right\|_{C B\left(\mathcal{S}^{p}\right)} \leqslant 1$ for $1<p<\infty$.

Remark 4.3. If $\mathbb{S}_{a \cdot b}$ acts on $\mathfrak{X}=B\left(\ell_{2}\right)$, one can prove the above lemma by using the version of Stinespring's Extension Theorem for restricted Schur multipliers developed in [19]. However, the above proof has the advantages of being constructive and of working for arbitrary unconditional matrix spaces.

Proof of Theorem 1.3. Recall that for any operator $T, a_{n}(T) \geqslant \max \left\{c_{n}(T), d_{n}(T)\right\}$, and a similar inequality holds for the c.b. versions of these $s$-numbers. On the other hand, by $\left[20, u_{n}(T) \leqslant c_{n}(T)\right.$ and $v_{n}(T) \leqslant d_{n}(T)$. Thus, Lemmas 4.2 and 4.1 provide the upper and lower estimates, respectively, for the $s$-numbers in question.

We have not been able to obtain sharp individual estimates for other $s$-sequences (see the next section for "collective" estimates). We did, however, obtain some results on the sequence of isomorphism numbers $i_{n}$. Recall that, for $T \in B(X, Y)$, we set $i_{n}(T)=0$ if $\operatorname{rank} T<n$. Otherwise, we set $i_{n}(T)=\sup \left\{\|A\|^{-1}\|B\|^{-1}\right\}$, where the supremum is taken over all spaces $G$ of dimension at least $n$ and all operators $A: G \rightarrow X$ and $B: Y \rightarrow G$ satisfying $B T A=I_{G}$.

Proposition 4.4. Suppose $\mathfrak{X}$ is an unconditional matrix space. Consider an operator $\mathbb{S}=\mathbb{S}_{a \cdot b}$ (with $a=\operatorname{diag}\left(a_{i}\right), b=\operatorname{diag}\left(b_{j}\right)$, where $a_{1} \geqslant a_{2} \geqslant \ldots>0$ and $\left.b_{1} \geqslant b_{2} \geqslant \ldots>0\right)$ as acting on $\mathfrak{X}$. Let $\lambda_{m}=\widetilde{[a b}_{m}(m \in \mathbb{N})$.

(1) There exist universal constants $c_{1}$ and $c_{2}$ such that, for any $n \in \mathbb{N}, i_{n}(\mathbb{S}) \geqslant$ $c_{1} \max \left\{\lambda_{\left\lceil c_{2} n(\log n+1)\right\rceil}, \lambda_{n} /(\log n+1)\right\}$.

(2) Moreover, if $\mathfrak{X}=\mathcal{S}^{\mathcal{E}}$, where the symmetric sequence space $\mathcal{E}$ has non-trivial Boyd indices, then $i_{n}(\mathbb{S}) \geqslant c \lambda_{n}$ ( $c$ is a constant depending on $\mathcal{E}$ ).

Proof. We first present (somewhat sketchily) the proof of part (1). As before, let $\mathbf{S}=\left\{(i, j): a_{i} b_{j} \geqslant \lambda_{n}\right\}$ and $E=\operatorname{span}\left[E_{i j}:(i, j) \notin \mathbf{S}\right]$. If the set $\mathbf{S}$ is infinite, then there exist $N, M \in \mathbb{N}$ s.t. $N M$ is arbitrarily large and $[1, N] \times[1, M] \subset \mathbf{S}$. Since the truncation projection onto the top left $N \times M$ corner is contractive, by Corollary 2.2 we conclude that $i_{n}(\mathbb{S}) \geqslant \lambda_{n}$ (this is exemplified by $G=\operatorname{span}\left[E_{k \ell}\right.$ : $1 \leqslant k \leqslant M, 1 \leqslant \ell \leqslant N]$ ).

Now suppose $\mathbf{S}$ is finite. For $i \in \mathbb{N}$, denote by $\tilde{j}_{i}$ the largest value of $j$ for which $(i, j) \in \mathbf{S}$. If there is no such $j$, set $\tilde{j}_{i}=0$. Then $\sum_{i=1}^{\infty} \tilde{j}_{i} \geqslant n$. We shall show that there exists $i$ s.t. $i \tilde{j}_{i} \geqslant \alpha n /(\log n+1)$, where $\alpha$ is a constant. Indeed, otherwise we would have

$$
n \leqslant \sum_{i} \tilde{j}_{i} \leqslant \frac{\alpha n}{\log n+1} \sum_{i=1}^{\lfloor\alpha n /(\log n+1)\rfloor} \frac{1}{i},
$$

and the right hand side is less than $n$ for sufficiently small values of $\alpha$. Estimating $i_{\lfloor\alpha n /(\log n+1)\rfloor}(\mathbb{S})$ using the space $G=\operatorname{span}\left[E_{k \ell}: 1 \leqslant k \leqslant i, 1 \leqslant \ell \leqslant \tilde{j}_{i}\right]$, we can show that

$$
i_{\lfloor\alpha n /(\log n+1)\rfloor}(\mathbb{S}) \geqslant a_{i} b_{\tilde{j}_{i}} \geqslant \lambda_{n} .
$$

Therefore, $i_{n}(\mathbb{S}) \geqslant c \lambda_{\left\lceil c_{2} n(\log n+1)\right\rceil}$, for some $c$. 
Next let $\mathbf{S}^{\prime}=\left\{(i, j): i \leqslant n, a_{i} b_{j} \geqslant \lambda_{n}\right\}$. This set has at least $n$ elements and can be written as $\bigcup_{k=1}^{\ell}\left[1, i_{k}\right] \times\left[1, j_{k}\right]$, with $\ell \leqslant n$. Then the "natural" truncation operator onto $\mathbf{S}^{\prime}$ has norm not exceeding $C(\log n+1)$, where $C$ is an absolute constant [16. Factoring the identity on $G=\operatorname{span}\left[E_{k \ell}:(k, \ell) \in \mathbf{S}^{\prime}\right]$, we see that $i_{n}(\mathbb{S}) \geqslant \lambda_{n} /(C(\log n+1))$.

To deal with part (2), suppose $\mathcal{E}$ has non-trivial Boyd indices. Then, by Section 4 of [3] (or Theorem 3.3 of [9]), the "natural" truncation onto $\mathbf{S}^{\prime}$ (viewed as an operator on $\mathcal{S}^{\mathcal{E}}$ ) has norm not exceeding some constant that depends only on $\mathcal{E}$.

\section{Membership IN OPERATOR IDEALS}

In this section, we consider the membership of elementary operators in operator ideals. We denote by $M_{A, B}$ the multiplication operator: if $X_{0}, X, Y, Y_{0}$ are Banach spaces, $A \in B\left(X_{0}, X\right)$, and $B \in B\left(Y, Y_{0}\right)$, we define $M_{A, B}: B(X, Y) \rightarrow B\left(X_{0}, Y_{0}\right)$ : $T \mapsto B T A$. Throughout this section, we assume that $A$ and $B$ are non-zero.

If $X_{0}=X=Y=Y_{0}=\ell_{2}, A=\operatorname{diag}(a), B=\operatorname{diag}(b)$, then $M_{A, B}=\mathbb{S}_{a \cdot b}$. If $\mathcal{E}$ is a symmetric sequence space and $A, B \in B\left(\ell_{2}\right)$, then $M_{A, B}^{\mathcal{E}}$ denotes the corresponding map from $\mathcal{S}^{\mathcal{E}}$ into itself.

In this section, we determine whether $M_{A, B}^{\mathcal{E}}$ belongs to a given operator ideal (see e.g. 22] for the terminology and the main results).

Proposition 5.1. Suppose $A$ and $B$ are operators between Hilbert spaces and that $2<p<\infty$. Suppose, furthermore, that $\mathcal{E}$ is a symmetric sequence space such that the formal identity $\ell_{2} \rightarrow \mathcal{E}$ is contractive. Then $\|A\|_{p}\|B\|_{p} \leqslant \pi_{p, 2}\left(M_{A, B}^{\mathcal{E}}\right) \leqslant$ $C_{p}\|A\|_{p}\|B\|_{p}$ (where $C_{p}$ is an absolute constant).

Denote by $x_{n}(T)$ the sequence of Weyl numbers of an operator $T \in B(X, Y)$ defined by

$$
x_{n}(T)=\sup \left\{a_{n}(T u): u \in B\left(\ell_{2}, X\right),\|u\| \leqslant 1\right\} .
$$

The quasi-Banach operator ideal $\mathfrak{L}_{p}^{(x)}$ consists of all operators $T$ for which the $\operatorname{sum} \sum_{n} x_{n}(T)^{p}$ is finite, equipped with the norm $\|T\|_{\mathfrak{L}_{p}^{(x)}}=\left(\sum_{n} x_{n}(T)^{p}\right)^{1 / p}$. See Section 2.4 of 23 for more information.

Suppose $A$ and $B$ are operators between Hilbert spaces, and consider the operator $M_{A, B}$ and its Weyl numbers. Note that if either $A$ or $B$ is non-compact (and both are non-zero), $M_{A, B}$ preserves a copy of $\ell_{2}$, hence $\lim \sup x_{n}\left(M_{A, B}\right)>0$.

Proposition 5.2. Suppose $A$ and $B$ are compact operators on a Hilbert space, $\mathcal{E}$ is a symmetric sequence space, and $1 \leqslant p<\infty$. Then

$$
c\|A\|_{p}\|B\|_{p} \leqslant\left(\sum_{n} x_{n}\left(M_{A, B}^{\mathcal{E}}\right)^{p}\right)^{1 / p} \leqslant\|A\|_{p}\|B\|_{p}
$$

(where $c$ is an absolute constant).

Proof. Suppose $T: X \rightarrow X$ is a compact operator with eigenvalues $\left(\lambda_{i}(T)\right)$, enumerated in such a way that $\left|\lambda_{1}(T)\right| \geqslant\left|\lambda_{2}(T)\right| \geqslant \ldots$. A Banach space version of the classical Weyl inequality (see e.g. [21]) shows that there exists an absolute constant $\kappa$ such that, for any $n \in \mathbb{N}$,

$$
\prod_{k=1}^{n}\left|\lambda_{k}(T)\right| \leqslant \kappa^{n} \prod_{k=1}^{n} \dot{x}_{k}(T), \text { where } \dot{x}_{k}(T)=x_{\lfloor(k+1) / 2\rfloor}(T) .
$$


A simple domination argument (see e.g. Chapter II of [5]) shows that $\left\|\left(\left|\lambda_{k}(T)\right|\right)\right\|_{\mathcal{F}} \leqslant$ $\kappa\left\|\left(\dot{x}_{k}(T)\right)\right\|_{\mathcal{F}} \leqslant 2 \kappa\left\|\left(x_{k}(T)\right)\right\|_{\mathcal{F}}$ for any symmetric sequence space $\mathcal{F}$.

In our situation, we may assume that $A=\operatorname{diag}\left(a_{i}\right)$ and $B=\operatorname{diag}\left(b_{j}\right)$. Then the eigenvalues of $M_{A, B}^{\mathcal{E}}$ are precisely the numbers $a_{i} b_{j}$. Then the left hand side of the lemma follows from the identity $\sum_{i, j}\left|a_{i} b_{j}\right|^{p}=\sum_{i}\left|a_{i}\right|^{p} \sum_{j}\left|b_{j}\right|^{p}$. To deal with the right hand side, observe that

$$
\sum_{n} x_{n}\left(M_{A, B}^{\mathcal{E}}\right)^{p} \leqslant \sum_{n} a_{n}\left(M_{A, B}^{\mathcal{E}}\right)^{p}=\sum_{i, j} a_{i}^{p} b_{j}^{p}=\|A\|_{p}\|B\|_{p},
$$

since, by Theorem 1.3, the sequence $\left(a_{n}\left(M_{A, B}^{\mathcal{E}}\right)\right)$ is a rearrangement of the double sequence $\left(a_{i} b_{j}\right)$.

Remark 5.3. In the same way, we can prove that when $\mathbb{S}_{a \cdot b}$ acts on an unconditional matrix space $\mathfrak{X}$, we have

$$
c\|a\|_{p}\|b\|_{p} \leqslant\left(\sum_{n} x_{n}\left(M_{A, B}^{\mathcal{E}}\right)^{p}\right)^{1 / p} \leqslant\|a\|_{p}\|b\|_{p}
$$

(where $c$ is an absolute constant). Here, $\|\cdot\|_{p}$ refers to the $\ell_{p}$ norm of a sequence.

Proof of Proposition 5.1. Note that if either $A$ or $B$ is not compact, then $M_{A, B}^{\mathcal{E}}$ preserves a copy of $\ell_{2}$ and hence doesn't belong to $\Pi_{p, 2}$. Thus, we can assume that $A=\operatorname{diag}(a)$ and $B=\operatorname{diag}(b)$, with $a=\left(a_{i}\right)$ and $b=\left(b_{j}\right) \in c_{0}$, have non-negative entries.

First we show that $\|A\|_{p}\|B\|_{p} \leqslant \pi_{p, 2}\left(M_{A, B}^{\mathcal{E}}\right)$. By definition,

$$
\left(\sum_{i, j}\left\|M_{A, B}^{\mathcal{E}} E_{i j}\right\|^{p}\right)^{1 / p} \leqslant \pi_{p, 2}\left(M_{A, B}^{\mathcal{E}}\right) \sup _{T \in \mathcal{S}^{\mathcal{E}^{*}},\|T\|_{\mathcal{E}^{*}} \leqslant 1}\left(\sum_{i, j}\left|\left\langle T, E_{i j}\right\rangle\right|^{2}\right)^{1 / 2} .
$$

But

$$
\sum_{i, j}\left\|M_{A, B}^{\mathcal{E}} E_{i j}\right\|^{p}=\sum_{i, j}\left|a_{i}\right|^{p}\left|b_{j}\right|^{p}=\|A\|_{p}^{p}\|B\|_{p}^{p} .
$$

On the other hand, $\left\langle T, E_{i j}\right\rangle=\operatorname{tr}\left(T^{t} E_{i j}\right)=T_{i j}$, and

$$
\sum_{i, j}\left|\left\langle T, E_{i j}\right\rangle\right|^{2}=\sum_{i, j}\left|T_{i j}\right|^{2}=\|T\|_{2}^{2} \leqslant\|T\|_{\mathcal{E}^{*}}^{2} \leqslant 1 .
$$

By (5.1), $\|A\|_{p}\|B\|_{p} \leqslant \pi_{p, 2}\left(M_{A, B}^{\mathcal{E}}\right)$.

To prove the opposite inequality, recall that by Theorem 17 of [21], for $2<p<\infty$ there exists a constant $\gamma_{p}$ s.t. $\pi_{p, 2}(T) \leqslant \gamma_{p}\left(\sum_{n} x_{n}(T)^{p}\right)^{1 / p}$ for every operator $T$. Applying Proposition 5.2, we complete the proof.

Remark 5.4. Some results related to those from Sections 4 and 5 need to be mentioned. By Proposition 3.3 of [6], for $1 \leqslant p<\infty, A \in B\left(X_{1}, X\right)$, and $B \in B\left(Y, Y_{1}\right)$, we have $\pi_{p}\left(M_{A, B}\right)=\pi_{p}\left(A^{*}\right) \pi_{p}(B)\left(M_{A, B}\right.$ is viewed as a map from $B(X, Y)$ to $\left.B\left(X_{1}, Y_{1}\right)\right)$. If $X, X_{1}, Y$, and $Y_{1}$ are Hilbert spaces, then $\pi_{p}\left(A^{*}\right) \sim\|A\|_{2}$ and $\pi_{p}(B) \sim\|B\|_{2}$, with equality for $p=2$ (see e.g. [22]). In this case, $\pi_{p}\left(M_{A, B}\right) \sim$ $\|A\|_{2}\|B\|_{2}$, with equality when $p=2$. The ideal norms and $s$-numbers of formal identity maps between non-commutative sequence spaces have been studied 
by A. Defant and his co-authors; see e.g. [8] and references therein. Finally, some estimates on the $s$-numbers of multiplication operators (and, more generally, of elementary operators) on $C^{*}$-algebras have recently been obtained in [2].

\section{REFERENCES}

1. A. Amir-Moez, Extreme properties of linear transformations and geometry in unitary spaces, Texas Tech University Press, Lubbock, TX, 1968. MR.0347865 (50:366)

2. M. Anoussis, V. Felouzis, and I. Todorov, S-numbers of elementary operators on $C^{*}$-algebras, preprint, J. Operator Theory, to appear.

3. J. Arazy, Some remarks on interpolation theorems and the boundedness of the triangular projection in unitary matrix spaces, Integral Equations Operator Theory, 1:453-495, 1978. MR.516764 (81k:47056a)

4. W. Banks and A. Harcharras, On the norm of an idempotent Schur multiplier on the Schatten class, Proc. Amer. Math. Soc., 132:2121-2125, 2004. MR2053985 (2005c:47047)

5. R. Bhatia, Matrix analysis, Springer-Verlag, New York, 1997. MR.1477662 (98i:15003)

6. B. Carl, A. Defant, and M. Ramanujan, On tensor stable operator ideals, Michigan Math. J., 36:63-75, 1989. MR989937 (90b:47080)

7. K. Davidson and A. Donsig, Norms of Schur multipliers, Illinois J. Math., 51:743-766, 2007. MR2379721 (2009k:47104)

8. A. Defant, M. Mastyło, and C. Michels, Summing norms of identities between unitary ideals, Math. Z., 252:863-882, 2006. MR2206631 (2006j:47035)

9. P. Dodds, T. Dodds, B. de Pagter, and F. Sukochev, Lipschitz continuity of the absolute value and Riesz projections in symmetric operator spaces, J. Funct. Anal., 148:28-69, 1997. MR 1461493 (98g:46098)

10. I. Doust and T. Gillespie, Schur multiplier projections on the von Neumann-Schatten classes, J. Operator Theory, 53:251-272, 2005. MR2153148 (2007b:47093)

11. E. Effros and Z.-J. Ruan, Operator spaces, The Clarendon Press, Oxford University Press, New York, 2000. MR1793753(2002a:46082)

12. I. C. Gohberg and M. G. KreĬn, Introduction to the theory of linear nonselfadjoint operators. American Mathematical Society, Providence, RI, 1969. MR0246142 (39:7447)

13. A. Harcharras, Fourier analysis, Schur multipliers on $S^{p}$ and non-commutative $\Lambda(p)$-sets, Studia Math., 137:203-260, 1999. MR1736011 (2001f:43004)

14. A. Harcharras, S. Neuwirth, and K. Oleszkiewicz, Lacunary matrices, Indiana Univ. Math. J., 50:1675-1689, 2001. MR1889075 (2003a:47047)

15. M. Hladnik, Compact Schur multipliers, Proc. Amer. Math. Soc., 128:2585-2591, 2000. MR 1766604 (2002a:46081)

16. S. Kwapień and A. Pełczyński, The main triangle projection in matrix spaces and its applications, Studia Math., 34:43-68, 1970. MR0270118 (42:5011)

17. T. Oikhberg, Direct sums of operator spaces, J. London Math. Soc. (2), 64:144-160, 2001. MR:1840776 (2002c:46115)

18. V. Paulsen, Completely bounded maps and operator algebras, Cambridge University Press, Cambridge, 2002. MR.1976867 (2004c:46118)

19. V. Paulsen, S. Power, and R. Smith, Schur products and matrix completions, J. Funct. Anal., 85:151-178, 1989. MR1005860 (90j:46051)

20. A. Pietsch, s-numbers of operators in Banach spaces, Studia Math., 51:201-223, 1974. MR $0361883(50: 14325)$

21. A. Pietsch, Weyl numbers and eigenvalues of operators in Banach spaces, Math. Ann., 247:149-168, 1980. MR.568205 (82i:47073a)

22. A. Pietsch, Operator ideals, North-Holland, Amsterdam, 1980. MR582655 (81j:47001)

23. A. Pietsch, Eigenvalues and s-numbers, Cambridge University Press, Cambridge, 1987. MR 890520 (88j:47022b)

24. G. Pisier, Multipliers and lacunary sets in non-amenable groups, Amer. J. Math., 117:337376, 1995. MR1323679 (96e:46078)

25. G. Pisier, Similarity problems and completely bounded maps. Second, expanded edition, Springer-Verlag, Berlin, 2001. MR 1818047 (2001m:47002)

26. G. Pisier, Introduction to operator space theory, Cambridge University Press, Cambridge, 2003. MR2006539 (2004k:46097) 
27. D. Potapov and F. Sukochev, Operator-Lipschitz functions in Schatten-von Neumann classes, preprint, available at http://xxx.lanl.gov/abs/0904.4095.

28. B. Simon, Trace ideals and their applications. Second edition, American Mathematical Society, Providence, RI, 2005. MR2154153 (2006f:47086)

Department of Mathematics, University of California - Irvine, Irvine, California 92697 - And - Department of Mathematics, University of Illinois at Urbana-Champaign, URBANA, ILLinois 61801

E-mail address: toikhber@math.uci.edu 